\title{
A Practical One-Pot Synthesis of Vinylstannanes from Ketones
}

\author{
Alla Darwish and J. Michael Chong* \\ Guelph-Waterloo Centre for Graduate Work in Chemistry and Biochemistry $\left(\mathrm{GWC}^{2}\right)$, \\ Department of Chemistry, University of Waterloo, Waterloo, Ontario, Canada \\ N2L 3G1 \\ jmchong@uwaterloo.ca \\ Supporting Information
}

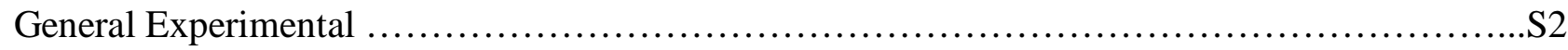

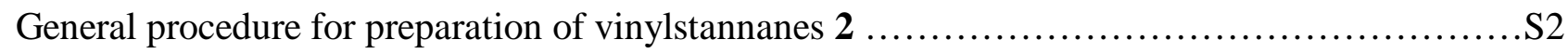

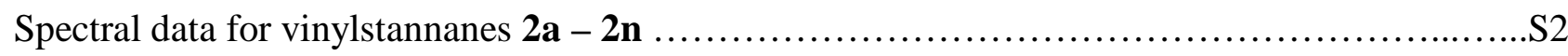

Spectral data for MOM ethers of alcohols from addition of $\mathrm{Bu}_{3} \mathrm{SnLi}$ to ketone $1 \mathbf{1}$...............S5

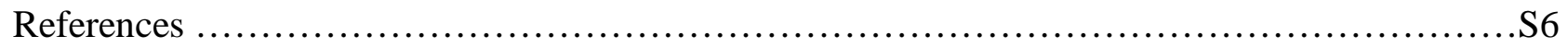




\section{$\underline{\text { General Experimental }}$}

All reactions were carried out under argon using flame-dried glassware. NMR data were recorded on $300 \mathrm{MHz}$ instruments in $\mathrm{CDCl}_{3}$ unless otherwise specified. Couplings to ${ }^{117} \mathrm{Sn}$ and ${ }^{119} \mathrm{Sn}$ are reported as averages of the two values. THF was distilled from Na/benzophenone, diisopropylamine was distilled from $\mathrm{CaH}_{2}$, and acetone was distilled from $\mathrm{B}_{2} \mathrm{O}_{3} \cdot \mathrm{Bu}_{3} \mathrm{SnH}$ was prepared from $\mathrm{Bu}_{3} \mathrm{SnOSnBu}_{3}$ and $\mathrm{NaBH}_{4} \cdot{ }^{1}$ Alkynyl ketones $\mathbf{1 h}$ and $\mathbf{1 i}$ were prepared by reaction of the appropriate alkynyllithium with dimethylacetamide. ${ }^{2} n$-BuLi was titrated using $N$-benzylbenzamide. ${ }^{3}$

General procedure for preparation of vinylstannanes 2 . To a cold $\left(0{ }^{\circ} \mathrm{C}\right)$, stirred solution of diisopropylamine (5 mmol, $1.0 \mathrm{eq})$ in THF (15 mL) was added $n$-BuLi (1.6 M in hexanes, $5 \mathrm{mmol}, 1.0$ eq). The solution was stirred at $0{ }^{\circ} \mathrm{C}$ for $10 \mathrm{~min}$ then $\mathrm{Bu}_{3} \mathrm{SnH}(5 \mathrm{mmol}, 1.0 \mathrm{eq})$ was added and the resulting yellow solution was stirred at $0{ }^{\circ} \mathrm{C}$ for an additional $10 \mathrm{~min}$. The solution was then cooled to $78{ }^{\circ} \mathrm{C}$ and the ketone (5 mmol, $\left.1.0 \mathrm{eq}\right)$ was added dropwise. After 10 minutes, $\mathrm{Et}_{3} \mathrm{~N}(40 \mathrm{mmol}, 8 \mathrm{eq})$ and $\mathrm{MsCl}$ (20 mmol, $4 \mathrm{eq})$ were added. The reaction mixture was then allowed to warm to rt. After 30 min at rt, hexanes $(200 \mathrm{~mL})$ was added and the organic layer was washed with $\mathrm{CH}_{3} \mathrm{CN}(3 \mathrm{x} 100 \mathrm{~mL})$. Concentration of the hexanes layer provided crude vinylstannanes. Traces of $\mathrm{Bu}_{3} \mathrm{SnH}$ could be removed by stirring the sample with a small amount of $\mathrm{AIBN}$ in $\mathrm{CHCl}_{3}(15 \mathrm{~mL}$ per gram of crude material $)$ at 40 ${ }^{\circ} \mathrm{C}$ for $4 \mathrm{~h}$ followed by flash chromatography on silica gel (10 g per gram of crude material). Alternatively, purification could be effected using flash column chromatography on reverse-phase silica gel ${ }^{4,5}$ using $40 \% \mathrm{CH}_{2} \mathrm{Cl}_{2}$ in $\mathrm{CH}_{3} \mathrm{CN}$. In all cases, vinylstannanes were isolated as colorless liquids.

1-Tributylstannylcyclohexene (2a). ${ }^{\mathbf{6 , 7 , 8 , 9 , 1 0} 1} \mathrm{H}$ NMR $\delta 5.78\left(1 \mathrm{H}, \mathrm{s}, \mathrm{J}_{\mathrm{Sn}-\mathrm{H}}=68.9 \mathrm{~Hz}\right), 2.70-2.50(4 \mathrm{H}$, m), $2.14-2.04(4 \mathrm{H}, \mathrm{m}), 1.54-0.82(27 \mathrm{H}, \mathrm{m})$

1-Tributylstannylcyclopentene (2b). ${ }^{7,9}{ }^{1} \mathrm{H}$ NMR $\delta 5.86\left(1 \mathrm{H}, \mathrm{t}, \mathrm{J}=2.1 \mathrm{~Hz}, \mathrm{~J}_{\mathrm{Sn}-\mathrm{H}}=35.2 \mathrm{~Hz}\right), 2.50-$ $2.28(2 \mathrm{H}, \mathrm{m}), 2.39-2.34(4 \mathrm{H}, \mathrm{m}), 1.58-0.86(27 \mathrm{H}, \mathrm{m})$ 
4-tert-Butyl-1-tributylstannylcyclohexene (2c). ${ }^{11}{ }^{1} \mathrm{H}$ NMR $\delta 5.78\left(1 \mathrm{H}, \mathrm{s}, \mathrm{J}_{\mathrm{Sn}-\mathrm{H}}=66.8 \mathrm{~Hz}\right), 2.31-$ $1.65(7 \mathrm{H}, \mathrm{m}), 1.55-0.84(36 \mathrm{H}, \mathrm{m}) ;{ }^{13} \mathrm{C}$ NMR $\delta \quad 140.0,137.5,44.1,33.7,32.4,29.4,29.3,27.5\left(\mathrm{~J}_{\mathrm{Sn}-\mathrm{C}}=\right.$ $60.4 \mathrm{~Hz}), 27.2,13.8,8.9\left(\mathrm{~J}_{\mathrm{Sn}-\mathrm{C}}=326.0 \mathrm{~Hz}\right)$

6-Methyl-1-tributylstannylcyclohexene (2d). ${ }^{9,10} \quad{ }^{1} \mathrm{H}$ NMR $\delta 5.73\left(1 \mathrm{H}, \mathrm{m}, \mathrm{J}_{\mathrm{Sn}-\mathrm{H}}=70.2 \mathrm{~Hz}\right), 2.24$ $(1 \mathrm{H}, \mathrm{m}), 2.00(2 \mathrm{H}, \mathrm{m}), 1.85-1.60(4 \mathrm{H}, \mathrm{m}), 1.57-0.82(30 \mathrm{H}, \mathrm{m}) ;{ }^{13} \mathrm{C} \mathrm{NMR} \delta 146.9,137.0,35.2,32.0$, $30.7,29.3,27.5\left(\mathrm{~J}_{\mathrm{Sn}-\mathrm{C}}=60.4 \mathrm{~Hz}\right), 23.0,20.5,13.8,9.8\left(\mathrm{~J}_{\mathrm{Sn}-\mathrm{C}}=339.6 \mathrm{~Hz}\right)$

1-Tributylstannyl-3,4-dihydronaphthalene (2e). ${ }^{1} \mathrm{H} \operatorname{NMR} \delta 7.11(3 \mathrm{H}, \mathrm{m}), 6.94(1 \mathrm{H}, \mathrm{d}, \mathrm{J}=7.2 \mathrm{~Hz})$, $6.23\left(1 \mathrm{H}, \mathrm{t}, \mathrm{J}=4.3 \mathrm{~Hz}, \mathrm{~J}_{\mathrm{sn}-\mathrm{H}}=59.6 \mathrm{~Hz}\right), 2.71(2 \mathrm{H}, \mathrm{t}, \mathrm{J}=7.8 \mathrm{~Hz}), 2.27(2 \mathrm{H}, \mathrm{m}), 1.55-0.84(27 \mathrm{H}, \mathrm{m})$; ${ }^{13} \mathrm{C}$ NMR $\delta 140.5,139.9,138.7,136.0,127.8,126.4,126.3,29.2,28.2,27.4\left(\mathrm{~J}_{\mathrm{Sn}-\mathrm{C}}=65.3 \mathrm{~Hz}\right), 24.8$, $13.8,10.1\left(\mathrm{~J}_{\mathrm{Sn}-\mathrm{C}}=326.3 \mathrm{~Hz}\right) ; \mathrm{IR}$ (neat) $2871,2853,1463,754 \mathrm{~cm}^{-1}$; MS $(\mathrm{EI}) \mathrm{m} / z 420\left(\mathrm{M}^{+}, 1 \%\right), 363$ $(\mathrm{M}-\mathrm{nBu}, 100 \%)$; Anal. Calcd for $\mathrm{C}_{22} \mathrm{H}_{36} \mathrm{Sn}: \mathrm{C}, 63.03 ; \mathrm{H}$, 8.66. Found: C, 63.00; H, 8.66.

1-Tributylstannyl-1-indene (2f). ${ }^{1} \mathrm{H}$ NMR $\delta 7.50(1 \mathrm{H}, \mathrm{d}, \mathrm{J}=7.2 \mathrm{~Hz}), 7.36(1 \mathrm{H}, \mathrm{d}, \mathrm{J}=7.4 \mathrm{~Hz}), 7.27$ $(1 \mathrm{H}, \mathrm{t}, \mathrm{J}=7.3 \mathrm{~Hz}), 7.17(1 \mathrm{H}, \mathrm{t}, \mathrm{J}=7.3 \mathrm{~Hz}), 6.68\left(1 \mathrm{H}, \mathrm{s}, \mathrm{J}_{\mathrm{Sn}-\mathrm{H}}=31.2 \mathrm{~Hz}\right), 3.46(2 \mathrm{H}, \mathrm{s}), 1.58-0.85(27 \mathrm{H}$, $\mathrm{m}) ;{ }^{13} \mathrm{C}$ NMR $\delta 150.0,145.0,144.8,143.9,126.2,124.2,123.6,122.4,41.5\left(\mathrm{~J}_{\mathrm{Sn}-\mathrm{C}}=48.3 \mathrm{~Hz}\right), 29.3$, $27.4\left(\mathrm{~J}_{\mathrm{Sn}-\mathrm{C}}=72.4 \mathrm{~Hz}\right), 13.8,9.5\left(\mathrm{~J}_{\mathrm{Sn}-\mathrm{C}}=350.2 \mathrm{~Hz}\right)$; IR (neat) $2871,2859,1604,1456,774 \mathrm{~cm}^{-1} ; \mathrm{MS}$ (EI) $m / z 406\left(\mathrm{M}^{+}, 0.7 \%\right), 349(\mathrm{M}-\mathrm{nBu}, 100 \%)$; Anal. Calcd for $\mathrm{C}_{21} \mathrm{H}_{34} \mathrm{Sn}$ : C, 62.25; H, 8.46. Found: C,62.20; H, 8.26.

1-Tributylstannyl-1-phenylethylene (2g). ${ }^{12}{ }^{1} \mathrm{H}$ NMR $\delta 7.31-7.16(5 \mathrm{H}, \mathrm{m}), 6.03(1 \mathrm{H}, \mathrm{d}, \mathrm{J}=2.4 \mathrm{~Hz}$, $\left.\mathrm{J}_{\mathrm{Sn}-\mathrm{H}}=130.6 \mathrm{~Hz}\right), 5.42\left(1 \mathrm{H}, \mathrm{d}, \mathrm{J}=2.4 \mathrm{~Hz}, \mathrm{~J}_{\mathrm{Sn}-\mathrm{H}}=60.8 \mathrm{~Hz}\right), 1.55-0.82(27 \mathrm{H}, \mathrm{m}) ;{ }^{13} \mathrm{C} \mathrm{NMR} \delta 154.7$, $146.5,128.3(2 \mathrm{C}), 126.8,126.4(2 \mathrm{C}), 126.3,29.1,27.4\left(\mathrm{~J}_{\mathrm{Sn}-\mathrm{C}}=60.4 \mathrm{~Hz}\right), 13.7,10.3\left(\mathrm{~J}_{\mathrm{Sn}-\mathrm{C}}=350.6 \mathrm{~Hz}\right)$

2-Tributylstannyl-dec-1-en-3-yne (2h). ${ }^{1} \mathrm{H}$ NMR $\delta 6.10\left(\mathrm{~d}, 1 \mathrm{H}, \mathrm{J}=3.3 \mathrm{~Hz}, \mathrm{~J}_{\mathrm{Sn}-\mathrm{H}}=117.8 \mathrm{~Hz}\right), 5.42$ $\left(\mathrm{d}, 1 \mathrm{H}, \mathrm{J}=3.4 \mathrm{~Hz}, \mathrm{~J}_{\mathrm{Sn}=\mathrm{H}}=55.2 \mathrm{~Hz}\right), 2.33(\mathrm{t}, 2 \mathrm{H}, \mathrm{J}=6.6 \mathrm{~Hz}), 1.55-0.85(\mathrm{~m}, 38 \mathrm{H}) ;{ }^{13} \mathrm{C} \mathrm{NMR} \delta 135.1$, $133.9,95.6,84.3,31.3,28.9,28.8,28.5,27.4\left(\mathrm{~J}_{\mathrm{Sn}-\mathrm{C}}=60.3 \mathrm{~Hz}\right), 22.5,19.7,13.9,13.6,10.0\left(\mathrm{~J}_{\mathrm{Sn}-\mathrm{C}}=338\right.$ $\mathrm{Hz}$ ); IR (neat) 2856, 1464, $668 \mathrm{~cm}^{-1}$; MS (EI) $\mathrm{m} / \mathrm{z} 426\left(\mathrm{M}^{+}, 0.5 \%\right), 369(\mathrm{M}-\mathrm{nBu}, 100 \%)$; Anal. Calcd for $\mathrm{C}_{22} \mathrm{H}_{42} \mathrm{Sn}$ : C, 62.13; H, 9.95. Found: C, 61.92; H, 9.89. 
2-Tributylstannyl-4-trimethylsilylbut-1-en-3-yne (2i). ${ }^{1} \mathrm{H}$ NMR $\delta 6.23\left(\mathrm{~d}, 1 \mathrm{H}, \mathrm{J}=3.3 \mathrm{~Hz}, \mathrm{~J}_{\mathrm{Sn}-\mathrm{H}}=\right.$ $112.5 \mathrm{~Hz}), 5.53\left(\mathrm{~d}, 1 \mathrm{H}, \mathrm{J}=3.3 \mathrm{~Hz}, \mathrm{~J}_{\mathrm{Sn}-\mathrm{H}}=54.0 \mathrm{~Hz}\right), 1.52-0.84(\mathrm{~m}, 27 \mathrm{H}), 0.15(\mathrm{~s}, 9 \mathrm{H},) ;{ }^{13} \mathrm{C} \mathrm{NMR} \delta$ 136.3, 134.7, 108.9, 99.3, 28.9, $27.4\left(\mathrm{~J}_{\mathrm{Sn}-\mathrm{C}}=57 \mathrm{~Hz}\right), 13.8,10.3\left(\mathrm{~J}_{\mathrm{Sn}-\mathrm{C}}=320 \mathrm{~Hz}\right), 0.2$; IR (neat) 2854, 2109, $842 \mathrm{~cm}^{-1}$; MS (EI) m/z $414\left(\mathrm{M}^{+}, 0.4 \%\right), 357(\mathrm{M}-\mathrm{nBu}, 100 \%)$; Anal. Calcd for $\mathrm{C}_{19} \mathrm{H}_{38} \mathrm{SiSn}: \mathrm{C}$, 55.21; H, 9.27. Found: C, $55.35 ; \mathrm{H}, 9.50$.

2-Tributylstannylpropene (2j). ${ }^{8}{ }^{1} \mathrm{H}$ NMR $\delta 5.67\left(1 \mathrm{H}, \mathrm{s}, \mathrm{J}_{\mathrm{Sn}-\mathrm{H}}=138.1 \mathrm{~Hz}\right), 5.07\left(1 \mathrm{H}, \mathrm{s}, \mathrm{J}_{\mathrm{Sn}-\mathrm{H}}=66.0\right.$ $\mathrm{Hz}), 1.95\left(3 \mathrm{H}, \mathrm{s}, \mathrm{J}_{\mathrm{Sn}-\mathrm{H}}=42.0 \mathrm{~Hz}\right), 1.56-0.85(27 \mathrm{H}, \mathrm{m}) ;{ }^{13} \mathrm{C} \mathrm{NMR} \delta 150.2,125.4\left(\mathrm{~J}_{\mathrm{Sn}-\mathrm{C}}=28.4 \mathrm{~Hz}\right)$, $29.0\left(\mathrm{~J}_{\mathrm{Sn}-\mathrm{C}}=19.9 \mathrm{~Hz}\right), 27.3\left(\mathrm{~J}_{\mathrm{Sn}-\mathrm{C}}=55.1 \mathrm{~Hz}\right), 13.5,9.0\left(\mathrm{~J}_{\mathrm{Sn}-\mathrm{C}}=321 \mathrm{~Hz}\right)$.

2-Tributylstannyl-3-methyl-1-butene (2k). ${ }^{8} \mathrm{H}$ NMR $\delta 5.66\left(1 \mathrm{H}, \mathrm{s}, \mathrm{J}_{\text {Sn-Htrans }}=143.1 \mathrm{~Hz}\right), 5.01(1 \mathrm{H}$, $\left.\mathrm{s}, \mathrm{J}_{\text {Sn-Hcis }}=66.0 \mathrm{~Hz}\right), 2.46(1 \mathrm{H}$, septet, $\mathrm{J}=6.8 \mathrm{~Hz}), 1.52-0.85(33 \mathrm{H}, \mathrm{m}) ;{ }^{13} \mathrm{C} \mathrm{NMR} \delta 162.5,121.7\left(\mathrm{~J}_{\mathrm{Sn}-\mathrm{C}}\right.$ $=28.5 \mathrm{~Hz}), 38.6\left(\mathrm{~J}_{\mathrm{Sn}-\mathrm{C}}=42.7 \mathrm{~Hz}\right), 29.0\left(\mathrm{~J}_{\mathrm{Sn}-\mathrm{C}}=21.0 \mathrm{~Hz}\right), 27.3\left(\mathrm{~J}_{\mathrm{Sn}-\mathrm{C}}=57.0 \mathrm{~Hz}\right), 23.0\left(\mathrm{~J}_{\mathrm{Sn}-\mathrm{C}}=14.8 \mathrm{~Hz}\right)$, $13.5,10.0\left(\mathrm{~J}_{\mathrm{Sn}-\mathrm{C}}=315.8 \mathrm{~Hz}\right)$.

4-Tributylstannyl-3,6-dihydro-2H-pyran (2l). ${ }^{13}{ }^{1} \mathrm{NMR} \delta 5.78\left(1 \mathrm{H}, \mathrm{m}, \mathrm{J}_{\mathrm{Sn}-\mathrm{H}}=64.3 \mathrm{~Hz}\right), 4.11(2 \mathrm{H}$, m), $3.74(2 \mathrm{H}, \mathrm{t}, \mathrm{J}=5.3 \mathrm{~Hz}), 2.24(2 \mathrm{H}, \mathrm{m}), 1.55-0.84(27 \mathrm{H}, \mathrm{m}) ;{ }^{13} \mathrm{C}$ NMR $\delta 137.7(\mathrm{Sn}-\underline{\mathrm{C}}), 135.9\left(\mathrm{~J}_{\mathrm{Sn}-\mathrm{C}}\right.$ $=18.0 \mathrm{~Hz}), 66.7\left(\mathrm{~J}_{\mathrm{Sn}-\mathrm{C}}=49.7 \mathrm{~Hz}\right), 64.6\left(\mathrm{~J}_{\mathrm{Sn}-\mathrm{C}}=27.7 \mathrm{~Hz}\right), 31.6\left(\mathrm{~J}_{\mathrm{Sn}-\mathrm{C}}=27.2 \mathrm{~Hz}\right), 29.0\left(\mathrm{~J}_{\mathrm{Sn}-\mathrm{C}}=19.9 \mathrm{~Hz}\right)$, $27.2\left(\mathrm{~J}_{\mathrm{Sn}-\mathrm{C}}=54.7 \mathrm{~Hz}\right), 13.6,8.7\left(\mathrm{~J}_{\mathrm{Sn}-\mathrm{C}}=325.4 \mathrm{~Hz}\right)$.

4-Tributylstannyl-3,6-dihydro-2H-thiopyran (2m). ${ }^{\mathbf{1 3}}{ }^{1} \mathrm{H}$ NMR $\delta 5.90\left(1 \mathrm{H}, \mathrm{m}, \mathrm{J}_{\mathrm{Sn}-\mathrm{H}}=66.9 \mathrm{~Hz}\right), 3.11$ $(2 \mathrm{H}, \mathrm{m}), 2.65(2 \mathrm{H}, \mathrm{t}, \mathrm{J}=5.6 \mathrm{~Hz}), 2.43(2 \mathrm{H}, \mathrm{m}), 1.48-0.82(27 \mathrm{H}, \mathrm{m}) ;{ }^{13} \mathrm{C} \mathrm{NMR} \delta 141.9\left(\mathrm{~J}_{\mathrm{Sn}-\mathrm{C}}=395.9\right.$ $\mathrm{Hz}), 132.4\left(\mathrm{~J}_{\mathrm{Sn}-\mathrm{C}}=25.5 \mathrm{~Hz}\right), 31.5\left(\mathrm{~J}_{\mathrm{Sn}-\mathrm{C}}=35.2 \mathrm{~Hz}\right), 28.9\left(\mathrm{~J}_{\mathrm{Sn}-\mathrm{C}}=19.7 \mathrm{~Hz}\right), 27.3\left(\mathrm{~J}_{\mathrm{Sn}-\mathrm{C}}=55.5 \mathrm{~Hz}\right)$, $26.4\left(\mathrm{~J}_{\mathrm{Sn}-\mathrm{C}}=58.1 \mathrm{~Hz}\right), 25.1\left(\mathrm{~J}_{\mathrm{Sn}-\mathrm{C}}=33.4 \mathrm{~Hz}\right), 13.6,8.8\left(\mathrm{~J}_{\mathrm{Sn}-\mathrm{C}}=323.0 \mathrm{~Hz}\right)$.

1-t-Butoxycarbonyl-4-Tributylstannyl-1,2,3,6-tetrahydropyridine (2n). ${ }^{13}{ }^{1} \mathrm{H} N \mathrm{NM} \delta 5.69(1 \mathrm{H}, \mathrm{s}$, $\left.\mathrm{J}_{\mathrm{Sn}-\mathrm{H}}=63.0 \mathrm{~Hz}\right), 3.86(2 \mathrm{H}, \mathrm{s}), 3.40(2 \mathrm{H}, \mathrm{t}, \mathrm{J}=5.4 \mathrm{~Hz}), 2.22(2 \mathrm{H}, \mathrm{s}), 1.42(9 \mathrm{H}, \mathrm{s}), 1.52-0.82(27 \mathrm{H}, \mathrm{m})$; ${ }^{13} \mathrm{C}$ NMR $\delta 154.8,138.7,133.4,79.1,45.1,39.8,39.0,31.5,28.9\left(\mathrm{~J}_{\mathrm{Sn}-\mathrm{C}}=19.9 \mathrm{~Hz}\right), 28.3,27.2\left(\mathrm{~J}_{\mathrm{Sn}-\mathrm{C}}=\right.$ $54.4 \mathrm{~Hz}), 13.5,8.9\left(\mathrm{~J}_{\mathrm{Sn}-\mathrm{C}}=325.5 \mathrm{~Hz}\right)$. 
Addition of $\mathrm{Bu}_{3} \mathrm{SnLi}$ to 2-methylcyclohexanone (1d). To examine the stereochemistry of addition of $\mathrm{Bu}_{3} \mathrm{SnLi}$ to ketone $\mathbf{1 d}$, an experiment was carried out in which the general procedure described on page $\mathrm{S} 2$ was followed but saturated aqueous $\mathrm{NH}_{4} \mathrm{Cl}$ was added instead of $\mathrm{MsCl} / \mathrm{Et}_{3} \mathrm{~N}$. The resulting unstable hydroxystannanes were isolated after standard aqueous workup and immediately treated with excess $\mathrm{MOMCl} / \mathrm{i}-\mathrm{Pr}_{2} \mathrm{NEt}$. Examination of the ${ }^{1} \mathrm{H}$ NMR spectrum of the crude reaction mixture indicated a 95:5 mixture of 2 diastereomers. These diastereomers were readily separated by flash chromatography on silica gel.

The major, more polar $\left(\mathrm{R}_{\mathrm{f}}=0.38\right.$, hexanes-ether, 10:1) isomer was assigned as the "axial" isomer based on data previously reported for the $\mathrm{Bu}_{3} \mathrm{SnLi}$ adducts to 4 -t-butylcyclohexanone $(\mathbf{1 c}){ }^{14}$ Of particular significance is ${ }^{3} \mathrm{~J}_{\mathrm{Sn}-\mathrm{C}}$ for $\mathrm{C} 3$ which is $\sim 0$ when the $\mathrm{Bu}_{3} \mathrm{Sn}$ group is axial and $\sim 40 \mathrm{~Hz}$ when the $\mathrm{Bu}_{3} \mathrm{Sn}$ group is equatorial, values consistent with a Karplus relationship.

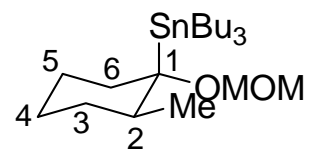

${ }^{1} \mathrm{H}$ NMR $\delta$ 4.70, $4.67\left(\mathrm{OC}_{2} \mathrm{O}, 2 \mathrm{H}, \mathrm{AB}\right.$ quartet, $\left.\mathrm{J}_{\mathrm{AB}}=6.9 \mathrm{~Hz}\right), 3.34\left(\mathrm{OCH}_{3}, \mathrm{~s}, 3 \mathrm{H}\right), 2.18(\mathrm{H} 6 \mathrm{eq}$, br d, $1 \mathrm{H}, \mathrm{J}=12.2 \mathrm{~Hz}), 1.73-0.85\left(\mathrm{CC}_{2} \underline{\mathrm{C}}_{2} \underline{\mathrm{CH}}_{2} \underline{\mathrm{CH}}_{2}, \mathrm{~m}, 7 \mathrm{H}\right),(\mathrm{Sn}-\underline{\mathrm{nBu}}, \mathrm{m}, 27 \mathrm{H}), 0.96\left(\mathrm{CHCH}_{3}, \mathrm{~d}, 3 \mathrm{H}, \mathrm{J}=\right.$ $6.6 \mathrm{~Hz}) ;{ }^{13} \mathrm{C}$ NMR $\delta 93.9\left(\mathrm{C} 1, \mathrm{~J}_{\mathrm{Sn}-\mathrm{C}}=435.4 \mathrm{~Hz}\right), 92.2\left(\mathrm{O}_{\underline{C}} \mathrm{H}_{2} \mathrm{O}, \mathrm{J}_{\mathrm{Sn}-\mathrm{C}}=24.7 \mathrm{~Hz}\right), 55.4\left(\mathrm{OC}_{3}\right), 41.6$ $\left(\mathrm{C} 2, \mathrm{~J}_{\mathrm{Sn}-\mathrm{C}}=26.0 \mathrm{~Hz}\right), 36.8\left(\mathrm{C} 6, \mathrm{~J}_{\mathrm{Sn}-\mathrm{C}}=31.7 \mathrm{~Hz}\right), 32.6\left(\mathrm{C} 3, \mathrm{~J}_{\mathrm{Sn}-\mathrm{C}} \sim 0 \mathrm{~Hz}\right), 29.3\left(\mathrm{SnCH}_{2} \mathrm{CH}_{2} \mathrm{CH}_{2} \mathrm{CH}_{3}, \mathrm{~J}_{\mathrm{Sn}-}\right.$ $\mathrm{C}=19.5 \mathrm{~Hz}), 27.7\left(\mathrm{SnCH}_{2} \mathrm{CH}_{2} \underline{\mathrm{CH}}_{2} \mathrm{CH}_{3}, \mathrm{~J}_{\mathrm{Sn}-\mathrm{C}}=59.4 \mathrm{~Hz}\right), 25.4\left(\mathrm{C} 5, \mathrm{~J}_{\mathrm{Sn}-\mathrm{C}} \sim 0 \mathrm{~Hz}\right), 24.9\left(\mathrm{C} 4, \mathrm{~J}_{\mathrm{Sn}-\mathrm{C}}=7.5\right.$ Hz) $20.2\left(\mathrm{CHCH}_{3}, \mathrm{~J}_{\mathrm{Sn}-\mathrm{C}}=19.5 \mathrm{~Hz}\right), 13.6\left(\mathrm{SnCH}_{2} \mathrm{CH}_{2} \mathrm{CH}_{2} \mathrm{CH}_{3}\right), 11.3\left(\mathrm{SnCH}_{2} \mathrm{CH}_{2} \mathrm{CH}_{2} \mathrm{CH}_{3}, \mathrm{~J}_{\mathrm{Sn}-\mathrm{C}}=282.7\right.$ $\mathrm{Hz})$

The minor, less polar $\left(\mathrm{R}_{\mathrm{f}}=0.49\right.$, hexanes-ether, 10:1) isomer was assigned as the "equatorial" isomer:

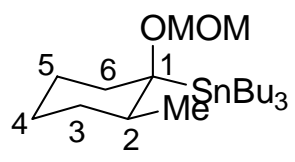


${ }^{1} \mathrm{H}$ NMR $\delta 4.66,4.57\left(\mathrm{OCH}_{2} \mathrm{O}, 2 \mathrm{H}, \mathrm{AB}\right.$ quartet, $\left.\mathrm{J}_{\mathrm{AB}}=6.6 \mathrm{~Hz}\right), 3.36\left(\mathrm{OC}_{3}, \mathrm{~s}, 3 \mathrm{H}\right), 2.26(\mathrm{H} 6 \mathrm{eq}$, br d, $1 \mathrm{H}, \mathrm{J}=11.5 \mathrm{~Hz}), 1.65-0.80\left(\mathrm{CC}_{2}{ }_{2} \underline{\mathrm{H}}_{2} \underline{\mathrm{CH}}_{2} \underline{\mathrm{CH}}_{2}, \mathrm{~m}, 7 \mathrm{H}\right),(\mathrm{Sn}-\underline{\mathrm{nBu}}, \mathrm{m}, 27 \mathrm{H}), 0.94\left(\mathrm{CHCH}_{3}, \mathrm{~d}, 3 \mathrm{H}, \mathrm{J}=\right.$ $6.6 \mathrm{~Hz}) ;{ }^{13} \mathrm{C}$ NMR $\delta 94.7\left(\mathrm{OC}_{2} \mathrm{O}, \mathrm{J}_{\mathrm{Sn}-\mathrm{C}}=19.2 \mathrm{~Hz}\right), 85.8(\mathrm{C} 1), 55.9\left(\mathrm{OCH}_{3}\right), 41.1(\mathrm{C} 2), 36.2\left(\mathrm{C} 6, \mathrm{~J}_{\mathrm{Sn}-\mathrm{C}}\right.$ $=21.7 \mathrm{~Hz}), 29.6\left(\mathrm{C} 3, \mathrm{~J}_{\mathrm{Sn}-\mathrm{C}}=34.7 \mathrm{~Hz}\right), 29.4\left(\mathrm{SnCH}_{2} \mathrm{CH}_{2} \mathrm{CH}_{2} \mathrm{CH}_{3}, \mathrm{~J}_{\mathrm{Sn}-\mathrm{C}}=19.3 \mathrm{~Hz}\right), 27.7$

$\left(\mathrm{SnCH}_{2} \mathrm{CH}_{2} \underline{\mathrm{CH}}_{2} \mathrm{CH}_{3}, \mathrm{~J}_{\mathrm{Sn}-\mathrm{C}}=57.4 \mathrm{~Hz}\right), 26.5(\mathrm{C} 4), 20.6\left(\mathrm{CHCH}_{3}, \mathrm{~J}_{\mathrm{Sn}-\mathrm{C}}=19.5 \mathrm{~Hz}\right), 20.4\left(\mathrm{C} 5, \mathrm{~J}_{\mathrm{Sn}-\mathrm{C}}=40.0\right.$ $\mathrm{Hz}), 13.6\left(\mathrm{SnCH}_{2} \mathrm{CH}_{2} \mathrm{CH}_{2} \underline{\mathrm{CH}_{3}}\right), 10.5\left(\mathrm{SnCH}_{2} \mathrm{CH}_{2} \mathrm{CH}_{2} \mathrm{CH}_{3}, \mathrm{~J}_{\mathrm{Sn}-\mathrm{C}}=280.9 \mathrm{~Hz}\right)$.

\section{$\underline{\text { References }}$}

${ }^{1}$ Szammer, J.; Otvos, L. Chem. Ind. 1988, 726.

${ }^{2}$ Brandsma, L. Preparative Acetylenic Chemistry, $2^{\text {nd }}$ ed; Elsevier: Amsterdam, 1988, p 103.

${ }^{3}$ Burchat, A. F.; Chong, J. M.; Nielsen, N. J. Organomet. Chem. 1997, 542, 281-283.

${ }^{4}$ Farina, V. J. Org. Chem. 1991, 56, 4985-4987.

${ }^{5} \mathrm{C} 18$ (reverse phase) silica is readily available but expensive. It can be made by a simple procedure (Evans, M.B. Chromatographia, 1980, 13, 5-10) and columns may be used multiple times.

${ }^{6}$ Gilbertson, S. R.; Challener, C. A.; Bos, M. E.; Wulff, W. D. Tetrahedron Lett. 1988, 29,4975-4798.

7 (a) Taylor,R. T.; Degenhardt, C. R.; Melega, W. P.; Paquette, L. A. Tetrahedron Lett. 1977, 159-162. (b) Adam, W.; Klug, P. J. Org. Chem. 1993, 58, 3416-3420. (c) von Zezschwitz, P.; Petry, F.; de Meijere, A. Chem. Eur. J. 2001, 7, 4035-4045. (d) Matsuura, A.; Komatsu, K. J. Am. Chem. Soc. 2001, 123, 1768-1769.

${ }^{8}$ Ratier, M.; Khatmi, D.; Duboudin, J. G.; Minh, D. T. Synth. Commun. 1989, 19, 285-291.

${ }^{9}$ Ratier, M.; Khatmi,, D.; Duboudin,J.. G. Bull. Soc. Chim. Belg. 1991, 100, 467-482.

${ }^{10}$ Duboudin, J. G.; Ratier, M.; Trouve, B. J. Organomet. Chem. 1987, 331, 181-191.

${ }^{11}$ Farina, V.; Kapadia, S.; Krishnan, B.; Wang, C.; Liebeskind, L. S. J. Org. Chem. 1994, 59, 59055911.

${ }^{12}$ Ritter, K. Synthesis 1989, 218-221.

${ }^{13}$ Quesnelle, C. A.; Gill, P.; Roy, S.; Dodier, M.; Marinier, A.; Martel, A.; Snyder, L. B.; D’Andrea, S. V.; Bronson, J. J.; Frosco, M.; Beaulieu, D.; Warr, G. A.; DenBleyker, K. L.; Stickle, T. M.; Yang, H.; Chaniewski, S. E.; Ferraro, C. A.; Taylor, D.; Russell, J. W.; Santone, K. S.; Clarke, J.; Drain, R. L.; Knipe, J. O.; Mosure, K.; Barrett,, J. F. Bio. Med.Chem. Lett. 2005, 15, 2728-2733.

${ }^{14}$ Sawyer, J. S.; Kucerovy, A.; Macdonald, T.; McGarvey, G. J. J. Am. Chem. Soc. 1988, 110, 842-853. 